\title{
An Improved Ant Colony Optimization for Multi-Depot Vehicle Routing Problem
}

\author{
Tang Yalian
}

\begin{abstract}
Considering multi-depot vehicle routing problem (MDVRP) is widely used in actual life, mathematical model of MDVRP was established. An improved ant colony optimization (IACO) was proposed for solving this model. Firstly, MDVRP was transferred into different groups according to nearest depot method, then constructing the initial route by scanning algorithm (SA). Secondly, genetic operators were introduced, and then adjusting crossover probability and mutation probability adaptively in order to improve the global search ${ }^{1}$ ability of the algorithm. Moreover, smooth mechanism was used to improve the performance of ant colony optimization (ACO). Finally, 3-opt strategy was used to improve the local search ability. The proposed IACO has been tested on 6 MDVRP benchmark problems. The experimental results show that IACO is superior to ACO in terms of convergence speed and solution quality, thus the proposed method is effective and feasible, and the proposed model is meaningful.
\end{abstract}

Index Terms-Smulti-depot vehicle routing problem, improved ant colony optimization, genetic algorithm, smooth mechanism.

\section{INTRODUCTION}

Multi-depot vehicle routing problem (MDVRP) is an extension of vehicle routing problem (VRP) and belongs to NP-hard. VRP has attracted high attention since it was proposed for the first time by Dantzig and Ramser in 1959 [1].With the rapid development of logistics, MDVRP is applied in kinds of industries, such as transportation, communications, industrial management, electric power and so on. VRP with multiple expansion characteristics are proposed in recent years [2]-[5]. Salhi, S, et al. [6] proposed an efficient implementation of variable neighborhood search that incorporates new features in addition to the adaptation of several existing neighborhoods and local search operators, in order to solve the multi-depot routing with heterogeneous vehicles. Alireza Rahimi-Vahed et al. [7] proposed a new Modular Heuristic Algorithm (MHA) whose exploration and exploitation strategies enable the algorithm to solve multi-depot periodic vehicle routing problem. Mehdi Adelzadeh et al. [8] proposed a mathematical model and a new heuristic solution method for solving multi-depot vehicle routing problem with time windows and different types of vehicles. This systematic approach has some steps as: customer clustering, routing, vehicle type determination, scheduling, and routes improvement using simulated annealing and customer service level improvement. In this

Manuscript received January 4, 2015. revised March 15, 2015.

Yalian Tang is with Dongguan Jinan University Institute, China (e-mail: tangyalian11@163.com). paper, a new improved ant colony optimization (IACO) is proposed for this model. The proposed IACO for MDVRP comprised three phases: constructing initial solution, global search and local search. The remainder of the paper was organized as follows. Section II stated the problem and mathematical model was established. Section III introduced the proposed IACO. The experimental results and analysis were reported in Section IV, followed by a summary and some concluding remarks in Section V.

\section{PROBlem Formulation}

In this section, we formally state the MDVRP, the ultimate goal is to decrease the cost of delivery and improve customer satisfaction. It is the key to determine the customers' service sequence. In addition, how to arrange the vehicle of the depot can affect the cost. In order to reduce the penalty cost, to give a reasonable arrangement for the departure time of vehicles is necessary. Customer demands are deterministic, the locations of customers and depots are known. The notations and mathematical formulation are as follows:

Sets:

$I:$ Set of all customers

$H:$ Set of all depots

$V:$ Set of all types of vehicles

Parameters:

$N$ : Number of all customers

$c_{i j}:$ The unit cost of transportation

$d_{i j}$ : Distance between point $i$

and $j, i, j \in I \cup H$

$g_{i}$ : Demand of customer $i$

$m_{r}^{h}:$ Number of routes in the

depot $h$

$k:$ The vehicle in the depot $h$

$M_{r}$ : Total number of routes to complete all tasks, $M_{r}=\sum_{h=1}^{H} m_{r}^{h}$

$Q(k)$ : Capacity of vehicle $k, h \in H, v \in V$

$C_{r}^{h}$ : Number of customers served on the route $r, C_{r}^{h} \geq 1, h \in H$

Decision variables:

$x_{i j k}: 1$, if vehicle $k$ finishes the task at customer $i$, then travels to $j ; 0$, otherwise.

$y_{i k}: 1$, if vehicle $k$ offer service for $i ; 0$, otherwise.

Mathematical model: 


$$
\begin{gathered}
\min z=\sum_{i \in I \cup H} \sum_{j \in I \cup H} \sum_{h \in H} d_{i j} c_{i j} x_{i j k} \\
\sum_{h \in H} \sum_{j \in I \cup H} x_{i j k}=1 \quad i \in I \\
\sum_{i \in I} g_{i} y_{i k} \leq Q(k) \\
\sum_{i \in I} \sum_{j \in H} x_{i j k} \leq 1 \quad h \in H, v \in V \\
\sum_{i \in I \cup H} x_{i j k}=\sum_{j \in I \cup H} x_{j i k} \leq 1 \quad i \in I \cup H, h \in H
\end{gathered}
$$

Eq. (1) is the objective function, the goal is to minimize the total cost. Eq. (2) makes sure that each customer is assigned to a single route. Eq. (3) is the capacity constraint set for vehicles. Eq. (4) assures that each customer can be served only once. Eq. (5) ensures that all delivery vehicles must return to the original depot after finishing the task.

\section{IMPROVED ANT COLONY OPTIMIZATION}

GA has rapid global search capability and handles multiple groups of individuals, but it doesn't make use of feedback information from the system, causing a lot of unnecessary redundancy iteration and leading to low solving efficiency ultimately. While ACO converges to the optimal path through pheromone accumulation and update, it has diversity and the positive feedback mechanism. However, it will cost too much time for accumulating pheromone in the early search process. Combining the advantages of the both algorithms, to construct a new IACO for MDVRP is our main work in this paper.

\section{A. Initialization}

SA (scanning algorithm) is proposed by Gillett and Miller in 1994[5]. The idea of the algorithm: taking the distribution center as an origin and grouping customers by rotating the ray, until it can't satisfy the constraint conditions, and then starting a new scanning, until all the customers are scanned on the plane.

\section{B. Genetic Algorithm}

\section{1) Encoding, fitness function and parameters design}

Integer encoding is used to encode the solution of the MDVRP. The fitness function is the reciprocal of the objective function. The parameters design including population size, denotes as $P o p_{\text {_ }}$ size, maximum iterations, denotes as Gen, crossover probability, denotes as $P_{c}$, mutation probability, denotes as $P_{m}$ and inversion probability, denotes as $P_{i n}$.

\section{2) Elite selection, crossover and mutation operation}

Elite selection is a kind of basic guarantee for converging to optimal solution. If the best individual adaptive value of the next population is less than the current best individual adaptive value, to copy the best individuals of the current population to the next generation, instead of the poor individuals.

Adopting the part map crossover, determining the parents' sample of crossover operation, and then the sample is grouped. To generate two random integers among $[1,10]$, determining two positions, to do a crossover operation. In the same way, do a mutation operation.

\section{3) Inversion operator}

Comparing crossover operator, inversion operator can make the offspring inherit more parental information. Supposing the code string is (0-1-3-4-7-8-2), there is a fracture between 3 and 4,8 and 2 , then do an inversion operation and insert, the new code string is (0-1-3-8-7-4-2). Because the inversion operation can make the evolutionary toward to the good direction, it is stronger than the crossover operator about optimization ability.

\section{Ant Colony Optimization}

\section{1) State transition rules}

Each ant, denotes as $\phi$, is assigned to a depot, the decision-making about combining customers is based on a probabilistic rule which taking into account of both the visibility and the pheromone information on an edge. The ant uses the formula (6) to select the next customer $\varphi$. Choosing probability between $\phi$ and $\varphi$ on the route is denoted by $P_{\phi \phi}^{k}$. Pheromone concentration on the edge $(\phi, \varphi)$ is denoted by $\tau_{\phi \varphi}$. The visibility on the edge $\tau_{\phi \varphi}$ is denoted by $\eta_{\phi \varphi}$. Pheromone trail is denoted by $\alpha$ and visibility value is denoted by $\beta$. The feasible customers' set is denoted by allowed $d_{k}$. Increasing the probability of random selection appropriately by combining deterministic selection and random selection in order to search the solution space more completely, which can overcome the defects of ACO. Eq. (8) is the rule. And $q$ is a random number among $[0,1], q_{0}$ is a parameter, and $q_{0} \in[0,1]$. Ant chooses the next customer as shown in the Eq. (7), or else chooses the edge as Eq. (8).

$$
P_{\phi \varphi}^{k}=\left\{\begin{array}{l}
\frac{\tau_{\phi \varphi}^{\alpha}(t) \eta_{\phi \varphi}^{\beta}(t)}{\sum_{\substack{\text { sallowed }_{k} \\
\text { otherwise }}}^{\alpha}(t) \eta_{\phi s}^{\beta}(t)}, \varphi \in \text { allowed }_{k} \\
0 \quad
\end{array}\right.
$$

$$
s=\left\{\begin{array}{l}
\arg \max _{s \in \text { allowed }_{k}}\left[\tau_{\phi s}^{\alpha}(t) \cdot \eta_{\phi s}^{\beta}(t)\right] \text { if }\left(q \leq q_{0}\right) \\
S, \quad \text { search probility according to } P_{\phi \varphi}^{k}(t)
\end{array}\right.
$$

\section{2) Updating pheromone information}

The pheromone increments are assigned to each visited edge. The pheromone updating equations are as Eq. (8), Eq. (9) and Eq. (10). The sum of pheromone increments on the edge $(\phi, \varphi)$ is denoted as $\Delta \tau_{\phi \varphi}$. The pheromone increment on edge $(\phi, \varphi)$ on the $k$ vehicle of the $\varphi$ depot is denoted as $\Delta \tau_{\phi \varphi}^{k \varphi}$. The parameter that controls the speed of evaporation is denoted as $\rho$. It can improve the global search ability by decrease $\rho$ but lower the convergence speed, so it's necessary to change $\rho$ adaptively by Eq. (11), and $\rho\left(t_{0}\right)=1$.

$$
\begin{gathered}
\tau_{\phi \varphi}(t+n)=\rho \cdot \tau_{\phi \varphi}(t)+(1-\rho) \cdot \Delta \tau_{\phi \phi} \\
\Delta \tau_{\phi \varphi}=\sum_{\varphi \in J} \sum_{k \in k} \Delta \tau_{\phi \varphi}^{k \varphi}
\end{gathered}
$$




$$
\begin{gathered}
\Delta \tau_{\phi \varphi}^{k \varphi}(t)=\left\{\begin{array}{l}
Q / d_{\phi \varphi k}, \text { if edge }(\phi, \varphi) \text { is serviced by } k \\
0, \text { otherwise }
\end{array}\right. \\
\rho(t)= \begin{cases}0.95 \rho(t-1) \quad \text { if } 0.95 \rho(t-1) \geq \rho_{\text {min }} \\
\rho_{\text {min }} & \text { otherwise }\end{cases}
\end{gathered}
$$

\section{3) Smooth mechanism}

Smooth mechanism is as shown in Eq. (12), $\delta \in(0,1)$, the pheromone concentration is denoted as $\tau_{\phi \phi}(t)$, and the pheromone track after smoothing is denoted as $\tau_{\phi \varphi}^{*}(t)$.

$$
\tau_{\phi p}^{*}(t)=\tau_{\phi p}(t)+\delta\left[\tau_{\max }(t)-\tau_{\phi p}(t)\right]
$$

\section{Adaptive Crossover Probability and Mutation} Probability

Crossover operator and mutation operator are introduced into ACO, crossover operator can increase the optimization space and mutation operator can restore the allele information which lost in evolution process, it can maintain the individual differences of population and prevent premature convergence by this way. On the other hand, mutation can improve the efficiency of local search for large-scale population. However, changing $P_{c}$ and $P_{m}$ as individual units is lack of team spirit, in some cases, this algorithm is not easy to escape from local optima, such as the stagnation of the overall evolution. Based on the above reasons, adjusting the crossover probability and mutation probability adaptively are necessary, as shown in Eq. (13) and Eq. (14), $P_{c}(t)$ and $P_{m}(t)$ are measured by average fitness $\left(f_{\text {ave }}\right)$, maximum fitness $\left(f_{\max }\right)$ and minimum fitness $\left(f_{\min }\right)$. The proximity of $\left(f_{\max }\right)$ and $\left(f_{\min }\right)$ reflects the concentration degree of the population, the closer, the more likely to fall into local optimal solution, and $0.5<a<1, P_{c}$ and $P_{m}$ hold the initial value.

$$
\begin{aligned}
& p_{c}(t)=\left\{\begin{array}{l}
p_{c} \cdot \frac{1}{1-\frac{f_{\min }}{f_{\max }}} \text {, when } \frac{f_{\text {ave }}}{f_{\max }}>a, \frac{f_{\min }}{f_{\max }}>b \\
p_{c}, \text { otherwise }
\end{array}\right. \\
& p_{m}(t)=\left\{\begin{array}{l}
p_{m} \cdot \frac{1}{1-\frac{f_{\min }}{f_{\max }}} \text {, when } \frac{f_{\text {ave }}}{f_{\max }}>a, \frac{f_{\min }}{f_{\max }}>b \\
p_{m}, \text { otherwise }
\end{array}\right.
\end{aligned}
$$

\section{E. 3-Opt Strategy}

3-opt strategy can enhance the search ability for ACO. The difference between 3-opt and 2-opt is that it uses two adjacent nodes, 3-opt is much more complex than 2-opt and the optimization effect is better. The steps are as follows: (1) Removing 3 edges from the path, and adding 3 edges in other parts, to form a full route. If the route length is shorter after the change, then keep the results; otherwise, trying other possible way by removing other edges and adding edges. (2) Repeat the above step, until tried to change all possible ways, and it can't improve the quality of the solution, then output the optimal solution, exit.

\section{F. The Best Fusion Time for GA and ACO}

1) Establishing the convergence efficiency evaluation function by objective function and fitness function.

2) To statistic the evolutionary rate of progeny groups in iterative process, denoted by $R$, $R=\{F(n+1)-F(n)\} / M_{f}\left(M_{f}\right.$ is a constant $)$, setting the minimum evolutionary rates of progeny groups, denoted by $R_{\min }$, and record the times for $R<R_{\min }$, denoted by Num .

3) If $N u m=T$ appears multiple times, $T$ is the set value, it means GA has been inefficient redundancy, then it's the time to start to ACO process.

\section{EXPERIMENTAL RESULTS AND ANALYSIS}

In this section, a complex MDVRP mathematical model has been set up. We performed some experiments on Microsoft Windows 7 operating systems, i5 core CPU Processor, 4G memory, 500G Hard disk, using Matlab R2010b software, in order evaluate the performance of the proposed algorithm, the performance of the algorithms was

\begin{tabular}{|c|c|c|c|c|c|c|}
\hline \multirow{3}{*}{ Sets } & \multicolumn{6}{|c|}{ Total cost } \\
\hline & \multicolumn{3}{|c|}{ IACO } & \multicolumn{3}{|c|}{$\mathrm{ACO}$} \\
\hline & Best & Average & Worst & Best & Average & Worst \\
\hline & 2.12 & 3.13 & 4.01 & 5.03 & 6.12 & 7.22 \\
\hline pro1 & 4.23 & 5.46 & 7.22 & 5.63 & 6.71 & 8.04 \\
\hline pro3 & 6.89 & 7.87 & 9.34 & 9.06 & 10.03 & 12.24 \\
\hline pr04 & 8.82 & 10.01 & 11.23 & 10.21 & 11.23 & 12.31 \\
\hline pro6 & 5.36 & 5.67 & 6.78 & 7.32 & 8.14 & 9.92 \\
\hline pro8 & 12.19 & 17.86 & 19.14 & 18.53 & 9.19 & 20.12 \\
\hline pro9 & 16.49 & 17.37 & 21.24 & 18.12 & 19.24 & 22.31 \\
\hline
\end{tabular}
tested on 6 benchmark data sets which can be downloading from the VRP Web at the website http://www.bernabe.dorronsoro.es/vrp/.

The parameters setting of IACO are as shown in Table I.

TABLE II: COMPARING RESULTS OF MDHVRPSTW WITH BKS ON MDVRPTW (\%)

\begin{tabular}{cccccccccc}
\hline Pop_size & Gen & $q_{0}$ & $Q$ & $\alpha=2$ & $\beta=2$ & $\rho_{\min }$ & $p_{c}$ & $p_{m}$ & $p_{\text {in }}$ \\
\hline 30 & 300 & 0.88 & 100 & 2 & 2 & 0.6 & 0.4 & 0.004 & 0.08 \\
\hline
\end{tabular}

The results of comparison between IACO and ACO based on 6 benchmark problems are summarized in Table II. We found that the performance of IACO is superior to ACO in terms of the convergence speed and the solutions' quality. This results attributes to the introduction of genetic operators, and changing crossover probability and mutation probability adaptively can improve the global search ability of the algorithm, moreover, 3-opt strategy can improve local search ability.

\section{CONCLUSIONS}

In this paper, an improved algorithm was proposed to solve MDVRP. This algorithm provides a feasible method for 6 benchmark problems. Considering VRP with a variety 
of characteristics and seeking new methods will be the future research direction.

\section{REFERENCES}

[1] R. A. Vahed, T. G. Crainic, M. Gendreau, and W. Rei, "A path relinking algorithm for a multi-depot periodic vehicle routing problem," Journal of Heuristics, vol. 19, no. 3, pp. 497-524, 2013

[2] W. Tu, Z. Fang, Q. Li, S. L. Shaw, and B. Y. Chen, "A bi-level Voronoi diagram-based metaheuristic for a large-scale multi-depot vehicle routing problem," Transportation Research Part E: Logistics and Transportation Review, vol. 61, pp. 84-97, 2014.

[3] S. Geetha, G. Poonthalir, and P. T. Vanathi, "Nested particle swarm optimisation for multi-depot vehicle routing problem," International Journal of Operational Research, vol. 16, no. 3, pp. 329-348, 2013.

[4] Y. Kuo and C. C. Wang, "A variable neighborhood search for the multi-depot vehicle routing problem with loading cost," Expert Systems with Applications, vol. 39, no. 8, pp. 6949-6954, 2012.

[5] B. Yao, P. Hu, M. Zhang et al., "Artificial bee colony algorithm with scanning strategy for the periodic vehicle routing problem," Simulation, vol. 89, no. 6, pp. 762-770, 2013
[6] S. Salhi, A. Imran, and N. A. Wassan, "The multi-depot vehicle routing problem with heterogeneous fleet: formulation and a variable neighborhood search implementation," Computers and Operations Research, vol. 52, pp. 315-325, 2014.

[7] A. R. Vahed, T. G. Crainic, M. Gendreau, and W. Rei, "Fleet-sizing for multi-depot and periodic vehicle routing problems using a modular heuristic algorithm," Computers and Operations Research, vol. 53, pp. 9-23, 2015.

[8] M. Adelzadeh, V. M. Asl, and M. Koosha, "A mathematical model and a solving procedure for multi-depot vehicle routing problem with fuzzy time window and heterogeneous vehicle," The International Journal of Advanced Manufacturing Technology, vol. 75, pp. 793802,2014

YaLian Tang was born in Hunan, China in 1986. She received her B.S degree in electronic information science and technology from Hunan University of Technology, HuNan, China, in 2008. Currently she is pursuing Ph.D degree in control science and engineering at Guangdong University of Technology. Her research interests include combinatorial optimization, intelligent algorithm and logistics control. 